\title{
Simple approach for ranking structure determining residues
}

Oscar D Luna-Martínez, Abraham Vidal-Limón, Miryam I Villalba-Velázquez, Rosalba Sánchez-Alcalá, Ramón RGJ Garduño-Juárez, Vladimir N Uversky, Baltazar Becerril

Mutating residues has been a common task in order to study structural properties of the protein of interest. Here, we propose and validate a simple method that allows the identification of structural determinants; i.e., residues essential for preservation of the stability of global structure, regardless of the protein topology. This method evaluates all of the residues in a 3D-structure of a given globular protein by ranking them according to their connectivity and movement restrictions without topology constraints. Our results matched up with sequence-based predictors that look up for intrinsically disordered segments, suggesting that protein disorder can also be described with the proposed methodology. 


\section{$1 \quad$ Simple Approach for Ranking Structure Determining Residues}

3 Oscar D. Luna-Martínez ${ }^{1}$, Abraham M. Vidal-Limón², Miryam I. Villalba-Velázquez ${ }^{1}$, Rosalba

4 Sánchez-Alcalá ${ }^{1}$, Ramón Garduño Juárez ${ }^{3}$, Vladimir N. Uversky ${ }^{4,5,6}$, and Baltazar Becerril ${ }^{1^{*}}$

5

$6{ }^{1}$ Instituto de Biotecnología, Universidad Nacional Autónoma de México, Cuernavaca, México

7 Apartado Postal 510-3

8 2División de Biología Molecular, Instituto Potosino de Investigación Científica y Tecnológica,

9 San Luis Potosí, México

10 32Instituto de Ciencias Físicas, Universidad Nacional Autónoma de México, Cuernavaca, México

11 Apartado Postal 48-3

$12{ }^{4}$ Department of Molecular Medicine and USF Health Byrd Alzheimer's Research Institute,

13 Morsani College of Medicine, University of South Florida, Tampa, FL, USA

$14{ }^{5}$ Institute for Biological Instrumentation, Russian Academy of Sciences, Pushchino, Moscow

15 Region, Russia

$16{ }^{6}$ Laboratory of Structural Dynamics, Stability and Folding of Proteins, Institute of Cytology,

17 Russian Academy of Sciences, St. Petersburg, Russia

18

$19 *$ Corresponding author

20

$21 *$ E-mail: baltazar@ibt.unam.mx

22

23 
24 Abstract. Mutating residues has been a common task in order to study structural properties of 25 the protein of interest. Here, we propose and validate a simple method that allows the 26 identification of structural determinants; i.e., residues essential for preservation of the stability of 27 global structure, regardless of the protein topology. This method evaluates all of the residues in a 28 3D-structure of a given globular protein by ranking them according to their connectivity and movement restrictions without topology constraints. Our results matched up with sequence-based predictors that look up for intrinsically disordered segments, suggesting that protein disorder can also be described with the proposed methodology.

\section{Introduction}

It is widely known that highly conserved residues in multiple sequence alignments of proteins are considered as key residues essential for protein structure and function including protein folding, phylogeny and evolutionary processes (Phillips, Janies \& Wheeler, 2000; Edgar \& Batzoglou, 2006; Pierri, Parisi \& Porcelli, 2010). Nevertheless, neutral mutations can accomplish great modifications to the structural properties of the protein by modifying its thermodynamic stability or biological function as seen in extremophiles in order to adapt to their environment (Jaenicke \& Böhm, 1998; Rothschild \& Mancinelli, 2001; Reed et al., 2013). Proteins can be considered as molecular entities with a tridimensional structure which are exposed to solvent and, frequently, interacting with other macromolecules. Each amino acid residue in a protein and each nucleotide base in a nucleic acid can be seen as a node and due to their high connectivity they represent hubs. Therefore, network analyses applied on protein structures attempt to assess the location of essential hubs that are necessary for the preservation 46 of the stability of the global structure (Vendruscolo et al., 2002). Several mathematical 
47 approaches have been described which have helped to identify these hubs, each one employing

48 different considerations such as geometrical approximations, thresholds, and information theory

49 (Costa et al., 2007; Böde et al., 2007). Other approaches combine network parameters with

50 protein properties, e.g. Relative Solvent Accesibility (RSA), to optimize the hierarchization of

51 each residue within the structure (Li, Wang \& Wang, 2008). In addition, the structural relevance

52 of each residue can be assessed by ranking the theoretical scores obtained from mathematical

53 approximations applied over protein structures (Greene \& Higman, 2003; Amitai et al., 2004).

54 Because protein structures are not rigid molecular assemblies, X-ray structure

55 determinations provide a "snapshot" of a "ground state", which is assumed to represent the

56 lowest energy conformation in a crystal lattice. (Karplus \& Kuriyan, 2005; Rodríguez-Rodríguez

57 et al., 2012). Molecular dynamics simulations is an in silico tool that can provide information on

58 detailed atomic motions at different time-scales, which have been increased through the

59 development of more powerful hardware (Kepleis et al., 2009; Dror et al., 2012). Taking

60 advantage of these technological advances, we sought to demonstrate that joining this important

61 parameter with network analyses will allow the compilation of a simple method for ranking

62 structure determining residues involved in protein stabilization. The problem of classifying each

63 node according to its structural relevance is far from being trivial because of various reasons. For

64 example, some residues that are not located at the hydrophobic core are known to have a long-

65 distance effect on the structure, even in the case of a neutral mutation (Tokuriki et al., 2008; Pace

66 et al., 2011). Our hypothesis is that the rigidness and the connectivity of each residue,

67 irrespectively of its solvent exposure, can be associated to a specific theoretical score that can be

68 used as a ranking parameter, where highly connected residues with restricted movement should

69 have the greatest effects on the overall stability of globular proteins. Among all network 
70 descriptors, we selected Shannon dynamical entropy as a connectivity parameter (Demetrius \&

71 Manke, 2004; Costa et al., 2007). In this study, some well-known protein structures were

72 unbiased chosen with the goal of developing a simple method to identify, in a hierarchical way,

73 those amino acid residues that are determinant in the structural stability of a protein. This

74 approach basically combines molecular dynamics with a network analysis based on the Shannon

75 dynamical entropy.

76

\section{Materials and Methods}

78 Statistics. Principal component analysis was performed using the R software (R Core Team, 79 2013). The thermal unfolding experiments of the 6aJL2 mutants were repeated in triplicate.

80 Crystallographic structures. The PDB codes, size, and protein class of the selected structures 81 are as follows: chymotrypsin inhibitor (2CI2; 65 residues; $16 \% \alpha+21 \% \beta)$; 6aJL2 (2W0K; chain

82 A, 111 residues; 5\% $\alpha+46 \% \beta$ ); apoflavodoxin (1FTG; 168 residues; 35\% $\alpha+19 \% \beta$ ); dimeric 83 Arc repressor (1ARR; 108 residues; 62\% $\alpha+9 \% \beta$ ); DNA-binding domain of human estrogen 84 receptor $\alpha$ in complex with the estrogen response element DNA duplex (1HCQ; chains A, B; 128 85 residues; $26 \% \alpha+9 \% \beta$, and chains C and D, 56 nucleotides); cold shock protein from Bacillus 86 subtilis (1CSP; 67 residues; 4\% $\alpha+55 \% \beta)$; cold shock protein from B. caldolyticus (1C9O, 66 87 residues; $4 \% \alpha+62 \% \beta$ ); and the largely disordered N-terminus of suppressor of cytokine 88 signaling 5 mammalian suppressor of Janus Kinase interaction region (2N34, 70 residues; 12\% 89 a). Minor structural modifications were performed on PDB files such as fulfilling N-terminal 90 domains and incomplete lateral chains by using Swiss PDB software (Guez \& Peitsch, 1997).

91 Furthermore, all nodes were renumbered in continuous order avoiding repetitions; i.e. in PDB 92 CI2 the first residue begins as residue 19. Consequently, all residues were systematically 
93 renumbered so the first residue was identified as residue 1 and so forth. Solvent-exposed area

94 was calculated with the NOC software (Chen, Cang \& Nymeyer, 2007).

95 Grade. Residues can be regarded as nodes and contacts as edges. Hence, an edge was defined 96 when any two non-hydrogen atoms from a pair of residues are within distance of $5 \AA$. To study

97 the topology of the residue contact network, we measured the degree of node- $i, \mathrm{~K} i$, as the 98 number of neighbors of node- $i$. Chain A of crystallographic structures was selected. In the case 99 of quaternary structures, all the structure was considered to measure the grade of each node but 100 only chain A was selected for further comparisons.

101 Molecular dynamics. The query protein was prepared using the Protein Preparation Wizard in 102 Maestro 9.2 package (Schrödinger LCC, NY) and included in a 10- $\AA$ water box that contained 10314513 SPC-type water molecules for 2CI2, 16667 for 6aJL2, 20051 for 1FTG, 20990 for 1ARR, 10428142 for 1HCQ, 12219 for 1CSP, and 12033 for 1C9O. Neutralizing ions were added, and other 105 metal ions already present in the protein structure were left at the same place. The simulated 106 annealing calculations and data analysis were conducted using the Desmond and Maestro 107 programs, respectively (Maestro-Desmond Interoperability Tools, version 3.0, Schrödinger, NY). 108 The OPLS_2005 force field was used for every molecular dynamics simulation. Cubic periodic 109 boundary conditions were used for most of the proteins $\left(53.9 \times 53.9 \times 53.9 \AA^{3}\right.$ for 2 CI2, $11056.8 \times 56.8 \times 56.8 \AA^{3}$ for $6 \mathrm{aJL} 2,60.9 \times 60.9 \times 60.9 \AA^{3}$ for $1 \mathrm{FTG}, 61.2 \times 61.2 .9 \times 61.2 \AA^{3}$ for $1 \mathrm{ARR}$, $11151.1 \times 51.1 \times 51.1 \AA^{3}$ for $1 \mathrm{CSP}$, and $50.8 \times 50.8 \times 50.8 \AA^{3}$ for $\left.1 \mathrm{C} 9 \mathrm{O}\right)$; due to the size of the 112 complex $1 \mathrm{HCQ}$, rectangular cuboid boundary conditions were used $\left(58.5 \times 59 \times 91 \AA^{3}\right.$ for $\left.1 \mathrm{HCQ}\right)$.

113 Each simulation was adjusted with an NPT ensemble by weak coupling to an external bath 114 temperature at constant pressure of $1 \mathrm{~atm}$ and relaxation time of $2 \mathrm{ps}$, regulated by Berendsen 115 barostat (Berendsen et al., 1984). All short-range interactions were computed using a $9 \AA$ cutoff, 
116 and for long-range interactions (electrostatic and van der Waals), a smooth particle mesh Ewald

117 method with a tolerance of $1 \times 10^{-9}$ was applied (Essmann et al., 1995). To ensure that our

118 simulations started from local minima, a simulated annealing algorithm was performed. This

119 method started the simulation at high temperature $(400 \mathrm{~K})$ to overcome thermodynamic and

120 conformational barriers, followed by gradual cooling (annealing) to reach low energy regimes. It

121 is widely used for the optimization of structures from experimental methods, comparative protein

122 modeling, or studying the conformational dynamics of protein or peptide folding and unfolding

123 (Mori et al., 2009). The full system was heated at $10 \mathrm{~K}$ for $30 \mathrm{ps,} 100 \mathrm{~K}$ for $100 \mathrm{ps,} 300 \mathrm{~K}$ for

$124200 \mathrm{ps}, 400 \mathrm{~K}$ for $300 \mathrm{ps}$, and $400 \mathrm{~K}$ for $500 \mathrm{ps}$ and then cooled to $298 \mathrm{~K}$ for $1000 \mathrm{ps}$. To ensure

125 that heating at $400 \mathrm{~K}$ did not affect protein or protein/DNA structure, the RMSD of heavy atoms

$126(\mathrm{C}, \mathrm{N}, \mathrm{O}, \mathrm{S}, \mathrm{P})$ derived from the annealed structure was compared against corresponding

127 crystallographic structure. If RMSD standard deviation was $\leq 1.0 \AA$, then it was assumed that the

128 annealing algorithm did not changed the whole structure or denature it. In fact, since the

129 displacement of heavy atoms above $1 \AA$ is considered as a conformational change, the algorithm

130 can be trusted in finding the local minimum. A lineal interpolation step between two adjacent

131 time points was employed. After the sixth step, a production of $25 \mathrm{~ns}$ was achieved with an

132 integration time of $1 \mathrm{fs}$. The entire analysis was performed using trajectory coordinates, and the

133 energies were written to a disk every 1.2 ps. A frame was extracted every 0.25 ns throughout the

134 simulation, and the overall frames were saved as a PDB file.

135 Dynamical entropy. An analytical algorithm encoded in the Perl language was developed to 136 generate three files using the 100-frames PDB-file as the template. In the first step, a single file

137 is generated for each model, and this single file contains the atom coordinates, the corresponding 138 molecular weight, and the name of the node (amino acid or nucleotide base). The second step 
139 calculates the coordinates of the center of mass of the node. The third step calculates the distance

140 between the center of mass of each pair of nodes and their normalized distance described by

141 Equation (1),

142

$$
\overline{d_{i j}}=\frac{d_{i j}}{r_{v d W_{i}}+r_{v d W_{j}}}
$$

143 where $\overline{d_{i j}}$ is the normalized distance, $d_{i j}$ is the distance between the center of mass of node $i$ and

144 that of node $j$, and $r_{v d W}$ is the van der Waals radius of the respective node considering all their

145 atoms. For amino acid residues, values of their van der Waals radiuses comprehending the whole

146 residue were obtained from Darby \& Creighton, 1993. For nucleotide bases, values were

147 obtained from Voss \& Gerstein, 2005. The mean distance between each pair of nodes of the 100

148 structures sampled was calculated. Since the next steps involve eigenvector and eigenvalue

149 measurement properties, the mean value of the inverse normalized distance was calculated, so

150 the largest weight represents the closest distance between a pair of nodes and the smallest weight

151 represents the longest distance.

152 A weighted adjacency matrix $\mathrm{A}=\left(a_{i j}\right) \geq 0$ of size $\mathrm{N} \times \mathrm{N}$, where $\mathrm{N}$ is the number of nodes, was 153 constructed. In this case, matrix $\mathrm{A}$ is symmetric $\left(a_{i j}=a_{j i}\right)$ and undirected. Following the 154 mathematical strategy described in Demetrius \& Manke, 2005, we now assume that the 155 stochastic process is given by a Markov Matrix $\mathrm{P}=p_{i j}$ where $p_{i j} \geq 0$ and $\Sigma_{j} p_{i j}=1$. The stationary 156 distribution of matrix $\mathrm{P}$ is described by Equation (2),

$$
\pi P=\pi
$$

158 where $\pi$ is defined as the left-hand eigenvector associated with the largest eigenvalue solved with

159 Mathcad $15^{\mathrm{TM}}$ software. The dynamical entropy of this process of each node, $\mathrm{H}_{\mathrm{i}}$, is described by 160 Equation (3), 
$161 \quad H_{i}=-\sum_{j} \pi_{i} p_{i j} \log p_{i j}$

162 where the term $p_{i j} \log p_{i j}$ is the standard Shannon entropy.

163 6aJL2 mutants. The synthesis of single-point mutants of 6aJL2 (Arg25His, Ile30Gly, Tyr36Phe, 164 and Gln6Asn) was performed using recursive PCR (Prodromou \& Pearl, 1992). The obtained 165 DNAs were cloned into the pSyn1 expression vector (Schier et al., 1995). All of the 166 constructions were verified by nucleotide sequencing (Sanger, Nicklen \& Coulson, 1977). The 167 variants were expressed in Escherichia coli BL21 (DE3) and purified as described previously 168 (del Pozo-Yauner et al., 2008). The protein purity was verified using SDS-PAGE 169 electrophoresis, and the protein concentration was determined spectrophotometrically at $280 \mathrm{~nm}$ 170 in $6.5 \mathrm{M} \mathrm{GdnHCl}$ and $20 \mathrm{mM}$ sodium phosphate buffer, $\mathrm{pH} \mathrm{7.5,} \mathrm{using} \mathrm{molar} \mathrm{extinction}$ 171 coefficients calculated from the amino acid sequence using the ProtParam software, which is 172 available at the ExPASy website (Gasteiger et al., 2005).

173 Unfolding. Samples containing $50 \mu \mathrm{g} / \mathrm{ml}$ of protein in phosphate-buffered saline (PBS), $\mathrm{pH} 7.5$, 174 were placed into a 3-ml quartz cuvette. Changes in the tryptophan fluorescence were measured 175 using a LS50B Perkin Elmer spectrofluorometer with an excitation wavelength of $295 \mathrm{~nm}(2.5$ $176 \mathrm{~mm}$ bandwidth) and an emission wavelength of $355 \mathrm{~nm}$ (5-mm bandwidth). The temperature was 177 increased from 298 to $350 \mathrm{~K}$ at a rate of $1 \mathrm{~K} / \mathrm{min}$ and then samples were cooled to $298 \mathrm{~K}$ at the 178 same rate. The data were analyzed using the thermal unfolding Equation (4), which was obtained 179 from Eftink (Eftink, 1995).

180

$$
F_{\text {Trp }}=\frac{\left(y_{n}+m_{n} T\right)+\left(y_{d}+m_{d} T\right) e^{\left(\frac{\Delta H_{m}}{R T_{m}}-\frac{\Delta H_{m}}{R T}\right)}}{1+e^{\left(\frac{\Delta H_{m}}{R T_{m}}-\frac{\Delta H_{m}}{R T}\right)}}
$$


181 where $F_{T r p}$ is the tryptophan fluorescence, $\mathrm{T}$ is the temperature, $T_{m}$ is the temperature of the

182 midpoint, $\Delta H_{m}$ is the enthalpy at $\mathrm{T}_{\mathrm{m}}, y_{n}$ and $m_{n}$ describe the pre-transition phase, and $y_{d}$ and

$183 m_{d}$ describe the post-transition phase. Non-linear regression was performed using the

184 OriginPro8 ${ }^{\mathrm{TM}}$ software. The change in the Gibbs energy of the wild-type versus the mutant $(\Delta \Delta G)$

185 was calculated for temperature- and denaturant-induced unfolding processes using the following 186 equations.

187 Thermal unfolding (Becktel \& Schellman, 1987), Equation (5):

188

$$
\Delta \Delta G=\frac{\Delta \mathrm{H}_{m W T}}{\mathrm{~T}_{m W T}}\left(T_{m M U T}-T_{m W T}\right)
$$

189 where WT refers to the wild-type values and MUT refers to the mutant values of the melting 190 temperature $\mathrm{T}_{\mathrm{m}}$, and $\Delta H_{m}$ is the enthalpy value. Chemical unfolding (Creighton, 1990), Equation (6):

$$
\Delta \Delta G=m_{W T}\left(C_{m M U T}-C_{m W T}\right)
$$

193 where $m_{W T}$ is the transition slope of the wild-type, and $\mathrm{C}_{\mathrm{m}}$ is the denaturant concentration at 194 which $\Delta G=0$. 


\section{Results and Discussion}

Many of the residues located at the hydrophobic core are considered essential (deleterious

198 if mutated), but it is hard to identify which one will be lethal through simple visual inspection of

199 a protein structure. In most cases reported so far, mutations increase the amount of unstable 200 conformers, rendering a protein more susceptible to external perturbations; and this is more

201 likely if the mutations are located at the inner core of the protein (Jackson et al., 1993; Baldwin 202 \& Matthews, 1994; Lei \& Duan, 2004; Kumar \& Nussinov, 2001; Reed et al., 2013).

203 Additionally, evaluating the role of each position by replacing the corresponding residue with 204 any other amino acid becomes a more challenging task as the protein becomes bigger. Therefore, 205 we sought to generate a simple method to assess proteins independently of their structural 206 complexity (secondary, tertiary or even quaternary structure).

We selected several proteins with diverse topologies that have been thermodynamically characterized to evaluate the effects of the incorporation of some single-point mutations near neutral pH. Chymotrypsin inhibitor, 6aJL2, apoflavodoxin, arc repressor, estrogen receptor/DNA estrogen /response element complex (ECR), cold shock protein of B. caldolyticus (Csp C), cold

211 shock protein of B. subtillis (Csp S), and the largely disordered N-terminus domain of Janus

212 Kinase interaction region were the representative proteins chosen for this study (see Methods

213 section for PDB details). Not only these proteins were selected because they exhibit different

214 folds, but also because they were analyzed using distinct experimental procedures that provided 215 different thermodynamic parameters covering two-state and three state unfolding pathways. 216 Most of the selected proteins have been thermodynamically evaluated elsewhere through 217 denaturation experiments using single-point mutants (Milla, Brown \& Sauer, 1994; Itzhaki, 218 Otzen \& Fersht, 1995; Perl \& Schmid, 2001; Banci et al., 2004; Campos et al., 2004a; Campos 
219 et al., 2004b; Wunderlich, Martin \& Schmid, 2005; Wunderlich \& Schmid, 2006; Gribenko \&

220 Makhatadze, 2007; del Pozo-Yauner et al, 2008; Deegan et al., 2010; Hernández-Santoyo et al.,

221 2010; van den Bedem et al., 2013; del Pozo-Yauner et al., 2014). $\Delta \Delta \mathrm{G}$ value represents the

222 effect of a determined mutation on the stability compared to the wild-type protein structure. On

223 the other hand, the estrogen receptor complex (ERC) was assessed by determining the affinity

224 constant $\left(\mathrm{K}_{\mathrm{D}}\right)$ against its target DNA. The percentage difference in terms of $\mathrm{K}_{\mathrm{D}}$ determines the

225 variation level in affinity between the wild-type ER homodimer and its mutant homodimer when

226 binding to its target DNA sequence. Both, percentages lower than 100 in $\mathrm{K}_{\mathrm{D}}$ and negative $\Delta \Delta \mathrm{G}$

227 values indicate less stable structures.

228 We compared these experimental data with a network parameter- Grade (see File S1).

229 This parameter is defined as the number of neighbors of each node when any two non-hydrogen

230 atoms from residues $i$ and $j$ are within a cutoff distance (Li, Wang, \& Wang, 2008). According to

231 Figure 1, there is a higher probability to affect the stability if the selected node is highly

232 surrounded in a cutoff distance of $5 \AA$. However, this parameter does not provide a full

233 hierarchization of each node; some nodes can share same grade-value but exhibit a different

234 impact on the stability. Another caveat is that size matters, because bigger residues, such as

235 tryptophan or phenylalanine, tend to make more interactions than smaller residues, such as

236 glycine or alanine (see File S1). Furthermore, proteins are not rigid structural assemblies since

237 most of their contacts are in a dynamical condition. Thus, we suggest that the molecular

238 dynamics of a protein should be gathered with more precise network analyses in order to

239 properly assess the influence of determined mutations on the stability of the protein structure.

As a first step to assess this assumption, the crystallographic structures were subjected to

$24125 \mathrm{~ns}$ of molecular dynamics simulations as described in the Methods section. The completion of 
242 this process was confirmed by inspecting the Root Mean Square Deviation (RMSD) values of the

243 main chain indicating the convergence to a stationary structural movement at $300 \mathrm{~K}$ (Figure 2).

244 Every $0.25 \mathrm{~ns}$, a structure was extracted by means of generating one hundred frames over all the

245 simulation time in order to obtain a good representation of the possible movements of both the

246 lateral and the main chains. Next, a network analysis was performed for each frame. In

247 accordance with definitions, each protein residue and each nucleotide base were considered as

248 individual nodes. The center of mass was calculated based on all of the atoms of the node. The

249 interaction strength between a pair of nodes was measured in terms of the distance between their

250 mass centers which was subsequently normalized by the summation of their van der Waals radii

251 considering all their atoms as stated in Materials and Methods section (values less than 1 imply

252 that the interaction is very strong).

253 We selected dynamical Shannon entropy as an approach to estimate connectivity being

254 aware that the results describe probabilistic values. The next step in the calculation process

255 included the use of eigenvector properties for which, the greater value, the more important

256 interaction. The inverse value of the mean normalized distance for the 100 frames was calculated

257 and rounded up to four decimals. At this point, we would like to emphasize that this strategy

258 makes a cutoff distance unnecessary. Then, each of these values was incorporated into a square

259 matrix that was, in turn, converted into a row stochastic matrix. Assuming that the microscopic

260 process of the network is Markovian, the matrix was solved consistently using a dynamical

261 entropy equation corresponding to a Markov process (Demetrius \& Manke, 2005; Costa et al.,

262 2007). Each node is now associated with a dynamical entropic value $\left(H_{i}\right)$, which can be

263 interpreted as a connectivity parameter with a probabilistic character. We also presume that the

264 movement restriction of each node, which is represented by its Root Mean Square Fluctuation 
265 (RMSF) value derived from the molecular dynamics, is associated with its dynamical entropic 266 value. Therefore, each node was scored by dividing its entropic value by its respective RMSF 267 value, and this score indicates the relative importance of each node within its respective structure 268 (see File S2). These theoretical scores were identified as structural-determining residue identifier 269 (SDRI). By normalizing SDRIs and plotting them against their sequence, SDRIs were distributed 270 throughout the structure (Figure 3). Distribution statistics- skewness, kurtosis, and Shapiro-Wilk 271 test showed that globular proteins are near normal distribution unlike the unstructured peptide 272 which is described hereinafter (Table 1). Interestingly, structures in complex - Arc repressor and 273 Complex Receptor DNA, showed fewer residues with low SDRI values compared to the other 274 analyzed structures, suggesting that both are notably less disordered.

275 We compared the distribution of the non-normalized SDRIs obtained in our study with 276 scores from the structure-based flexibility as well as the sequence-based intrinsic disorder 277 predisposition of the query proteins. The structural flexibility of these proteins was obtained by 278 utilizing the FlexPred tool that predicts the absolute fluctuations per-residue from a 279 three-dimensional structure using the B-factors of a query protein (Jamroz et al., 2012). The 280 intrinsic disorder propensities per-residue of these proteins was obtained by using PONDR ${ }^{\circledR}$ 281 VSL2B predictor, which is one of the more accurate stand-alone disorder predictors (Fan \& 282 Kurgan 2014; Peng et al., 2005; Peng \& Kurgan 2012). Results of these comparisons are shown 283 in Figure 4 and clearly illustrated that these three computational tools, SDRI, FlexPred, and 284 PONDR ${ }^{\circledR}$ VSL2B, can "see" different, although related, features in a protein. Note that we used 285 (1-SDRI) function instead of SDRI when representing the SDRI values to compare data from 286 these three tools "in phase" to highlight intrinsic disorder instead of structural rigidness. These 287 results suggested that there is a good agreement between the structural flexibility calculated from 
288 the protein crystal structure and the propensity of a protein to preserve disorder. Furthermore, it 289 seemed that residues essential for the preservation of the stability of global protein structure are 290 typically located within highly ordered and less flexible domains. In terms of spectral analysis, 291 the visual inspection of plots shown in Figure 4 suggested that, in many cases, the propensity for 292 intrinsic disorder results in broad bands that define global appearance of the curves, whereas the 293 outputs of (1-SDRI) and FlexPred add fine structural resolution to the resulting plots. 294 Additionally, for some regions, noticeable disagreements can be found among the outputs of 295 these three tools, which can be attributed to the particular considerations of each tool. 296 Nevertheless, these important observations suggested that intrinsic disorder propensity, predicted 297 from amino acid sequence, serves as an important background defining global flexibility of a 298 protein 3D-structure which is fine-tuned by long-distance interactions taking place in a folded 299 molecule.

A more efficient way to exploit SDRIs was to visualize each node according to its 301 theoretical score; the higher SDRI the more important is the residue to preserve the structure. In 302 homodimeric domains (arc repressor and ERC) both monomers showed the same distribution. In 303 our analysis, the residues with highest SDRI values corresponded to structure-determinant 304 residues (Figure 5). Complementarily, heat maps based on these values facilitated the 305 localization of essential residues or segments involved in a protein's biological function (Figure 306 6). Despite that linear statistics parameters (R-squared and Pearson Correlation Coefficient) 307 showed low correlation between SDRIs and experimental values (Table 2), visual inspection of 308 Figure 5 strongly suggested that a pattern was followed. Therefore, we performed a statistical 309 scrutiny applying Principal Component Analyses (PCA) to identify data patterns of apparently 310 uncorrelated variables (Abdi \& Williams, 2010). Results were represented as circular biplots 
311 allowing us to examine the correlation between variables SDRI and experimental data as vectors

312 (see Figure 5 and Supplemental Figure 2). Based on biplots from PCA results and due to the

313 angle between variables SDRIs and thermodynamic data, it can be emphasized that the present

314 method is able to estimate the effect of a single-point mutation on protein structure depending on

315 the importance of a given residue irrespective of its position. The results confirmed our

316 hypothesis that the most connected and the most rigid residues are the most influential on the

317 structural stability of the protein despite their involvement in any kind of structural organization.

318 For example, the worst effects in Arc repressor were observed in five mutants (VA22, EA36,

319 IA37, VA41, and FA45) that showed little or no cooperation in denaturation experiments (Milla,

320 Brown \& Sauer, 1994). Remarkably, these mutations were performed on residues that matched

321 with high SDRI values $(6,4,1,2$, and 19 , respectively).

322 The next comparison was between the SDRIs of two structures that have different

323 thermodynamic stability in spite of their high sequence and high structural homology. We

324 selected the Cold shock protein from thermophilic B. subtilis (Csp S) and from

325 hyperthermophilic B. caldolyticus (Csp C) bacteria. Csp C and Csp S share a sequence homology

$326>80 \%$, but the hyperthermophilic variant, Csp C, is more stable than its thermophilic counterpart.

327 Perl and Schmidt generated mutants in Csp S by directing them to Csp C sequence (see Figure

328 S1) (Perl \& Schmid, 2001). Our results show that Csp S stabilizing mutations were performed

329 over low connected and highly flexible residues, residues with low SDRIs. Most stabilizing

330 mutations of Csp S, that were directed to the sequence of the hyperthermophilic variant- Csp C,

331 were those incorporated on the surface bonding flexible residues through the formation of salt

332 bridges. Likewise, Tokuriki and Tawfik reported that mutations on surface residues of their

333 analyzed proteins resulted in low destabilizing effects while mutations on core residues caused 
334 stronger destabilizing effects (Tokuriki \& Tawfik, 2009).

335 We are particularly interested in the characterization of 6aJL2, an immunoglobulin light

336 chain variable domain, based on the fact that $6 a$ is the most implicated germ line in AL

337 amyloidosis disease (Comenzo et al., 2001). Destabilizing mutations of 6aJL2 enhances its

338 propensity to generate protein fibers. Strikingly, the crystallographic structures of destabilizing

339 mutants exhibited a low RMSD difference when overlapped against the wild-type structure

340 (Hernández-Santoyo et al., 2010). To experimentally demonstrate our hypothesis, we selected

341 four residues with low RSA values but different SDRIs to perform single-point mutations:

342 Gln6Asn, Arg24His, Tyr36Phe, and Ile29Gly (SDRIs 0.5055, 0.3273, 0.3274, and 0.2057,

343 respectively). Despite that the greatest destabilization impact was detected when the size of the

344 lateral chain was minimized, as observed with mutant Ile29Gly (RSA $2.2 \%, \Delta \Delta \mathrm{G}=-3.61 \mathrm{kcal}$

$\left.345 \mathrm{~mol}^{-1}\right)$, mutant Gln6Asn (RSA $12 \%, \Delta \Delta \mathrm{G}=-3.34 \mathrm{kcal} \mathrm{mol}^{-1}$ ) was more relevant due to the

346 neutrality of the change. The lateral chain size-reduction by one methylene had a remarkable

347 impact on the protein stability. We selected residue Gln6 in 6aJL2 because it is a highly

348 conserved residue in immunoglobulin light chains sequence alignments (Williams et al., 1996).

349 Opposite to this neutral mutation, Tyr36Phe did not affect the structure suggesting that the

350 hydroxyl group of the tyrosine is not playing a relevant role (RSA $0.6 \%, \Delta \Delta \mathrm{G}=-0.04 \mathrm{kcal}^{\mathrm{mol}}{ }^{-}$

$351{ }^{1}$ ). While $\operatorname{Arg} 24 \mathrm{His}\left(\operatorname{RSA} 10 \%, \Delta \Delta \mathrm{G}=-2.70 \mathrm{kcal} \mathrm{mol}^{-1}\right.$ ) performed in this work was more

352 destabilizing than reported mutation Arg24Gly $\left(\Delta \Delta \mathrm{G}=-1.52 \mathrm{kcal} \mathrm{mol}^{-1}\right)$ (del Pozo et al, 2008),

353 other effects should be considered like Phe2 reorientation to the upper hydrophobic core to

354 compensate the absence of the Arg24 guanidinium group (del Pozo et al, 2014). Interestingly,

355 Phe2 is not among the residues with higher SDRIs in 6aJL2 pointing out that compensatory

356 effects might be attributable to the flexibility of the lateral chain. 
358 SDRIs. Since proteins can interact with other proteins and other macromolecular, important 359 residues not only maintain the connectivity along the tertiary structure but also maintaining 360 quaternary structure as seen in the complex of the estrogen receptor with DNA by modifying the 361 affinity (Deegan et al., 2010). We found that the most flexible and unconnected regions were 362 associated with active site functions, as in the case of apoflavodoxin, to which the cofactor, 363 flavin mononucleotide, binds (Genzor et al., 1996). In chymotrypsin inhibitor, the larger loop, 364 which is flexible and unconnected, harbors the active site (Jackson et al, 1993). However, not all 365 mutations can enhance the stability while preserving the original function of a protein. In the 366 case of T4 lysozyme, some mutants were found to be more stable but resulted in losses of the 367 protein's original function (Shoichet et al., 1995). If the purpose is to modify the function of a 368 protein, potential mutations should be assessed by other means, such as evolutionary multiple 369 sequence alignment (Alexander et al., 2009; Halabi et al., 2009). If the aim is only to increase 370 the protein stability, a good approach could be locating the less structurally important residues 371 and generating changes that benefit the formation of salt bridges as shown in apoflavodoxin.

372 Mutations localized on the surface and designed to establish salt bridges were able to increase 373 the overall stability in apoflavodoxin (Campos et al., 2004). Remarkably, these mutations were 374 performed on the low-scored SDRI residues.

375 Since folding requires certain flexibility degree, we analyzed the relationship between 376 kinetic data with SDRIs. Other experiments performed in chymotrypsin inhibitor were 377 folding/unfolding kinetics (Figure 7) (Itzhaki, Otzen \& Fersht, 1995). In this case, the SDRIs 378 showed a better correlation with the $\Delta \Delta \mathrm{G}$ unfolding kinetic values ( $75 \%$ from the PCA analysis) 379 than the $\Delta \Delta \mathrm{G}$ folding kinetic values (57\% from the PCA analysis). We would like to reiterate 
380 that folding is a dynamic rearrangement of the network because longer times and other

381 conditions are required for better simulations of the protein folding/unfolding pathways.

Our last validation was performed over an intrinsically disordered peptide, resolved by

383 NMR in solution, which only showed a small structured portion of the peptide (Chandrashekaran

384 et al, 2015). Before applying the network strategy described here, the RMSD of each one of the

38520 frames was calculated by comparing them against the first frame (Figure 8A). It was evident

386 that there is a remarkable structural motion freedom at the N-terminus, even higher than those

387 obtained by molecular dynamics of the other proteins described here (Figures 2 , 4, and 8B). In

388 order to follow the methodology proposed here, RMSF values of the main chain of each residue

389 were calculated using RCI server (Berjanskii \& Wishart, 2013). An important difference

390 compared to globular proteins was the distribution statistics of the SDRIs, having the highest

391 value dispersion dissimilar to a normally distributed population (Table 1, Figure 4H). The most

392 connected and less flexible residues were located in the hairpin, shown in Figure 8C, which 393 contributes to the scaffolding functionality allowing phosphorylation of Ser211. Also, the

394 plasticity of the disordered N-terminus would enable this peptide to bind multiple components of

395 the signaling pathway in which it is involved (Chandrashekaran et al, 2015).

396 It should be noticed that applying this methodology on proteins with high structural

397 motion might not provide enough information to predict which residues will interact with

398 ligands. Unstructured peptides can be analyzed by other means (Kosol et al., 2013; Shaw et al., 399 2010). We simulated the unbound estrogen receptor and, despite the drastic change on the 400 ranking position and the SDRIs, it is not evident which segments of the estrogen receptor dimer 401 will recognize precise DNA sequence (see File S4). Furthermore, we decided to extend 6aJL2 402 simulation time to $50 \mathrm{~ns}$ and we only detected subtle, but not significant, changes on SDRIs for 
403 this globular protein (see File S4). Thus, appropriate molecular dynamics simulation 404 accomplished on globular proteins is an essential step for this methodology. Moreover, our 405 results agreed with sequence-based predictors that look up for the intrinsically disordered 406 segments regardless protein complexity. SDRI values displayed a versatile mathematical 407 parameter since function (1-SDRI) might be applicable to highlight disordered segments. Such 408 disordered segments may increase the capability of organisms to tolerate environmental 409 challenges by diversifying the properties of their proteins to recognize several molecular partners 410 such as cofactors, DNA, or other proteins. Higher SDRI values imply higher probabilities to 411 modify protein stability, but there is a higher tendency to destabilization. As reported by

412 Tokuriki and Tawfik, 2009, one of the evolutionary implications of protein destabilization is that 413 other functions or adaptations may be achieved. Finally, under the scenario of analyzing 414 uncommon foldings or even structures bearing non-natural amino acids, our method might be 415 suitable to assess structure stability since it does not depend on previous information such as an 416 evolutionary multiple sequence alignment. In conclusion, we have validated a method for the 417 analysis of globular proteins by ranking each one of their residues according to their structural 418 relevance from a theoretical score- SDRI.

420 Acknowledgements. We thank J. Osuna-Quintero, J.L. Martínez-Morales, and M. Aldana421 González for the valuable discussions and advice, C. Torres-Duarte for critically reviewing the 422 manuscript, the Oligonucleotide Synthesis Unit of the Institute of Biotechnology, T. Olamendi423 Portugal for the DNA sequencing, and G. Corzo-Burguete for facilitating the use of the computer 424 hardware that was used for the molecular dynamics simulations 


\section{References}

427 Abdi H, Williams LJ. 2010. Principal component analysis. WIREs Computational Statistics 428 2:433-459. DOI: $10.1002 /$ wics. 101

429 Alexander PA, He Y, Chen Y, Orban J, Bryan PN. 2009. A minimal sequence code for switching 430 protein structure and function. Proceedings of the National Academy of Sciences of the United 431 States of America 106:21149-21154. DOI: 10.1073/pnas.0906408106

432 Amitai G., Shemesh A, Sitbon E, Shklar M, Netanely D, Venger I, Pietrokovski S. 2004. 433 Network analysis of protein structures identifies functional residues. Journal of Molecular 434 Biology 344:1135-1146. DOI:10.1016/j.jmb.2004.10.055

435 Baldwin EP, Matthews BW. 1994. Core-packing constraints, hydrophobicity and protein design. 436 Current Opinion Biotechnology 5:396-402. DOI: 10.1016/0958-1669(94)90048-5

437 Banci L, Bertini I, Calderone V, Cramaro F, Del Conte R, Fantoni, Mangani S, Quattrone A, 438 Viezzoli MS. 2004. A prokaryotic superoxide dismutase paralog lacking two Cu ligands: From 439 largely unstructured in solution to ordered in the crystal. Proceedings of the National Academy of 440 Sciences of the U.S.A. 102:7541-7546. DOI: 10.1073/pnas.0502450102

441 Becktel WJ, Schellman JA. 1987. Protein stability curves. Biopolymers 26:1859-1877. DOI: 442 10.1002/bip.360261104

443 Berendsen HJC, Postma JPM, van Gunsteren WF, DiNola A, Haak JR. 1984. Molecular 444 dynamics with coupling to an external bath. The Journal of Chemical Physics 81: 3684-3690 445 DOI: $10.1063 / 1.448118$

446 Berjanskii MV, Wishart DS. 2013. A simple method to measure protein side-chain mobility 447 using NMR chemical shifts. Journal of the American Chemical Society 135: 14536-14539. DOI: $448 \quad 10.1021 / \mathrm{ja} 407509 \mathrm{z}$ 
449 Böde C, Kovács IA, Szalay MS, Palotai R, Korcsmáros T, Csermely P. 2007. Network analysis 450 of protein dynamics. FEBS Letters 581:2776-2782. DOI: 10.1016/j.febslet.2007.05.021

451 Campos LA, Bueno M, Lopez-Llano J, Jiménez MA, Sancho J. 2004a. Structure of Stable 452 Protein Folding Intermediates by Equilibrium $\phi$-Analysis: The Apoflavodoxin Thermal 453 Intermediate. Journal of Molecular Biology 344:239-255. DOI: 10.1016/j.jmb.2004.08.081

454 Campos LA, Garcia-Mira MM, Godoy-Ruiz R, Sanchez-Ruiz JM, Sancho J. 2004b. Do Proteins 455 Always Benefit from a Stability Increase? Relevant and Residual Stabilization in a Three-state 456 Protein by Charge Optimization. Journal of Molecular Biology 344:223-237. DOI: 457 10.1016/j.jmb.2004.09.047

458 Chandrashekaran IR, Mohanty B, Linossi EM, Dagley LF, Leung EWW, Murphy JM, Babon JJ, 459 Nicholson SE, Norton RS. 2015. Structure and functional characterization of the conserved JAK 460 interaction region in the intrinsically disordered N-Terminus of SOCS5. Biochemistry 54:4672461 4682. DOI: 10.1021/acs.biochem.5b00619

462 Chen ME, Cang HX, Nymeyer H. Dr. 2007. Nymeyer's Group, Inst. Mol. Biol., Florida State 463 University. Available online at http://noch.sourceforge.net (accessed 27 Nov 2015).

464 Comenzo RL, Zhang Y, Martinez C, Osman K, Herrera GA. 2001. The tropism of organ 465 involvement in primary systemic amyloidosis: contributions of $\operatorname{Ig} \mathrm{V}(\mathrm{L})$ germ line gene use and 466 clonal plasma cell burden. Blood 98:714-720. DOI: 10.1182/blood.V98.3.714

467 Costa LF, Rodrigues FA, Travieso G, Villas Boas PR 2007. Characterization of complex 468 networks: A survey of measurements. Advances in Physics 56:167-242. DOI: $46910.1080 / 00018730601170527$

470 Creighton TE. 1990. Protein structure: a practical approach. Oxford, England. IRL Press. 
471 Darby NJ, Creighton TE. 1993. Protein structure. Oxford: IRL Press at Oxford University Press. 472 p. 4. ISBN $019963310 \mathrm{X}$

473 Deegan BJ, Seldeen KL, McDonald CB, Bhat V, Farooq A. 2010. Binding of the ER $\alpha$ Nuclear 474 Receptor to DNA Is Coupled to Proton Uptake. Biochemistry 49:5978-5988. DOI: $47510.1021 / \mathrm{bi} 1004359$

476 del Pozo-Yauner L, Ortiz E, Sánchez R, Sánchez-López R, Güereca L, Murphy CL, Allen A, 477 Wall JS, Fernández-Velasco DA, Solomon A, Becerril B. 2008. Influence of the germline 478 sequence on the thermodynamic stability and fibrillogenicity of human lambda 6 light chains. 479 Proteins Structure Function and Bioinformatics 72:684-692. DOI: 10.1002/prot.21934

480 del Pozo-Yauner L, Wall JS, Gonzalez AM, Sanchez-López R, Rodriguez-Ambriz, Pérez481 Carreón JI, Ochoa-Leyva A, Fernández-Velasco DA. 2014. The N-terminal strand modulates 482 immunoglobulin light chain fibrillogenesis. Biochemical and Biophysical Research 483 Communications 443:495-499. DOI: 10.1016/j.bbrc.2013.11.123

484 Demetrius L, Manke T. 2004. Robustness and network evolution—an entropic principle. Physica 485 A 346:682-696. DOI: 10.1016/j.physa.2004.07.011

486 Dror RO, Dirks RM, Grossman JP, Xu H, Shaw DE. 2012. Biolomolecular simulation: A 487 computational microscope for molecular biology. Annual Review of Biophysics 41:429-452. 488 DOI: 10.1146/annurev-biophys-042910-155245

489 Edgar RC, Batzoglou S. 2006. Multiple sequence alignment. Current Opinion in Structural 490 Biology 16:368-373. DOI: 10.1016/j.sbi.2006.04.004

491 Eftink MR. 1995. Use of multiple spectroscopic methods to monitor equilibrium unfolding of 492 proteins. Methods in Enzymology 259:487-512. DOI: 10.1016/0076-6879(95)59058-7 
493 Essman U, Perera L, Berkowitz ML, Darden T., Lee H., Pedersen LG. 1995. A smooth particle

494 mesh Ewald method. The Journal of Chemical Physics 103:8577-85933. DOI:

$49510.1063 / 1.470117$

496 Fan X, Kurgan L. 2014. Accurate prediction of disorder in protein chains with a comprehensive 497 and empirically designed consensus. Journal of Biomolecular Structure and Dynamics 32:448498 464. DOI: $10.1080 / 07391102.2013 .775969$

499 Gasteiger E, Hoogland C, Gattiker A, Duvaud S, Wilkins MR, Appel RD, Bairoch A. 2005. 500 Protein Identification and Analysis Tools on the ExPASy Server. The Proteomics Protocols 501 Handbook. Humana Press. pp. 571-607. DOI: 10.1385/1-59259-890-0:571

502 Genzor CG, Perales-Alcón A, Sancho J, Romero A. 1996. Closure of a tyrosine/tryptophan 503 aromatic gate leads to a compact fold in apoflavodoxin. Nature Structural \& Molecular Biology 504 3:329-332. DOI: 10.1038/nsb0496-329

505 Greene LH, Higman VA. 2003. Uncovering network systems within protein structures. Journal 506 of Molecular Biology 334:781-791. DOI: 10.1016/j.jmb.2003.08.061

507 Gribenko AV, Makhatadze GI. 2007. Role of the Charge-Charge Interactions in Defining 508 Stability and Halophilicity of the CspB Proteins. Journal of Molecular Biology 366:842-856.

509 DOI: $10.1016 /$ j.jmb.2006.11.061

510 Guex N, Peitsch MC. 1997. SWISS-MODEL and the Swiss-PdbViewer: An environment for 511 comparative protein modeling. Electrophoresis 18:2714-2723. DOI: 10.1002/elps.1150181505

512 Halabi N, Rivoire O, Leibler S, Ranganathan R. 2009. Protein sectors: evolutionary units of 513 three-dimensional structure. Cell 138:774-786. DOI: 10.1016/j.cell.2009.07.038

514 Hernández-Santoyo, A. del Pozo Yauner L, Fuentes-Silva D, Ortiz E, Rudiño-Piñera E, Sánchez515 López R, Horjales E, Becerril B, Rodríguez-Romero A. 2010. A single mutation at the sheet 
516 switch region results in conformational changes favoring lambda6 light-chain fibrillogenesis.

517 Journal of Molecular Biology 396:280-292. DOI: 10.1016/j.jmb.2009.11.038

518 Itzhaki LS, Otzen DE, Fersht AR. 1995. The structure of the transition state for folding of 519 chymotrypsin inhibitor 2 analysed by protein engineering methods: evidence for a nucleation520 condensation mechanism for protein folding. Journal of Molecular Biology 254:260-288. DOI:

$521 \quad 10.1006 /$ jmbi.1995.0616

522 Jackson SE, Moracci M, elMasry N, Dobson CM, Fersht AR. 1993. Effect of cavity-creating 523 mutations in the hydrophobic core of chymotrypsin inhibitor 2. Biochemistry 32:11259-11269.

524 DOI: 10.1021/bi00093a001

525 Jaenicke R, Böhm G. 1998. The stability of proteins in extreme environments. Current Opinion 526 in Structural Biology 8:738-748. DOI: 10.1016/S0959-440X(98)80094-8

527 Jamroz M, Kolinski A, \& Kihara D. 2012. Structural features that predict real-value fluctuations 528 of globular proteins. Proteins: Structure, Function, and Bioinformatics 80:1425-1435. DOI: $52910.1002 /$ prot. 24040

530 Karplus M, Kuriyan J. 2005. Molecular dynamics and protein function. Proceedings of the 531 National Academy of Sciences of the United States of America 102:6679-6685. DOI:

$53210.1073 /$ pnas.0408930102

533 Kepleis JL, Lindorff-Larsen K, Dror RO, Shaw DE. 2009. Long-timescale molecular dynamics 534 simulations of protein structure and function. Current Opinion in Structural Biology 19:120-127 535 DOI: $10.1016 /$ j.sbi.2009.03.004

536 Kosol S, Contreras-Martos S, Cedeño C, Tompa P. 2013. Structural characterization of 537 intrinsically disordered proteins by NMR spectroscopy. Molecules 18:10802-10828. DOI: $538 \quad 10.3390 /$ molecules 180910802 
539 Kumar S, Nussinov R. 2001. How do thermophilic proteins deal with heat? Cellular and

540 Molecular Life Sciences 58:1216-1233. DOI: 10.1007/PL00000935

541 Lei H, Duan Y. 2004. The role of plastic $\beta$-hairpin and weak hydrophobic core in the stability 542 and unfolding of a full sequence design protein. The Journal of Chemical Physics 121:12104-

543 12111. DOI: $10.1063 / 1.1822916$

544 Li J, Wang J, Wang W. 2008. Identifying folding nucleus based on residue contact networks of 545 proteins. Proteins: Structure, Function, and Bioinformatics 71: 1899-1907. DOI: $546 \quad 10.1002 /$ prot.21891

547 Milla ME, Brown BM, Sauer RT. 1994. Protein stability effects of a complete set of alanine 548 substitutions in Arc repressor. Nature Structural \& Molecular Biology 1:518-523. DOI: $549 \quad 10.1038 / \mathrm{nsb} 0894-518$

550 Mori T, Okamoto Y. 2009. Folding simulations of gramicidin A into the $\beta$-helix conformations: 551 Simulated annealing molecular dynamics study. The Journal of Chemical Physics 131:165103. 552 DOI: $10.1063 / 1.3247578$

553 Pace CN, Fu H, Fryar KL, Landua, J, Trevino, SR, Shirley BA, McNutt Hendricks M, Iimura S, 554 Gajiwala K, Scholtz JM, Grimsley GR. 2011. Contribution of Hydrophobic Interactions to 555 Protein Stability. Journal of Molecular Biology 408:514-528. DOI: 10.1016/j.jmb.2011.02.053

556 Peng K, Vucetic S, Radivojac P, Brown CJ, Dunker AK, \& Obradovic Z. 2005. Optimizing long 557 intrinsic disorder predictors with protein evolutionary information. Journal of Bioinformatics 558 and Computational Biology 3:35-60. DOI: 10.1142/S0219720005000886

559 Peng ZL, and Kurgan L. 2012. Comprehensive comparative assessment of in-silico predictors of 560 disordered regions. Current Protein \& Peptide Science 13:6-18. DOI: $561 \quad 10.2174 / 138920312799277938$ 
562 Perl D, Schmid FX. 2001. Electrostatic stabilization of a thermophilic cold shock protein.

563 Journal of Molecular Biology 313:343-357. DOI: 10.1006/jmbi.2001.5050

564 Phillips A, Janies D, Wheeler W. 2000. Multiple sequence alignment in phylogenetic analysis.

565 Molecular Phylogenetics and Evolution 16:317-330. DOI: 10.1006/mpev.2000.0785

566 Pierri CL, Parisi G, Porcelli V. 2010. Computational approaches for protein function prediction:

567 A combined strategy from multiple sequence alignment to molecular docking-based virtual 568 screening. Biochimica et Biophysica Acta 1804:1695-1712. DOI: 10.1016/j.bbapap.2010.04.008

569 Prodromou C, Pearl LH. 1992. Recursive PCR: a novel technique for total gene synthesis. 570 Protein Engineering 5:827-829. DOI: 10.1093/protein/5.8.827

571 R Core Team. R: A language and environment for statistical computing Vienna, Austria. 2013. 572 ISBN: 3-900051-07-0. Available online at http://R-project.org (accessed 27 Nov 2015).

573 Reed CJ, Lewis H, Trejo E, Winston V, Evilia C. 2013. Protein adaptaptations in archaeal 574 extremophiles. Archaea Article ID 373275. DOI: 10.1155/2013/373275

575 Rodríguez-Rodríguez ER, Ledezma-Candanoza LM, Contreras-Ferrat LG, Olamendi-Portugal T, 576 Possani LD, Becerril B, Riaño-Umbarila L. 2012. A single mutation in framework 2 of the heavy 577 variable domain improves the properties of a diabody and a related single chain antibody. 578 Journal of Molecular Biology 423:337-350. DOI: 10.1016/j.jmb.2012.07.007

579 Rothschild LJ, Mancinelli RL. 2001. Life in extreme environments. Nature 409:1092-1101 DOI: $580 \quad 10.1038 / 35059215$

581 Sanger F, Nicklen S, Coulson AR. 1977. DNA sequencing with chain-terminating inhibitors. 582 Proceedings of the National Academy of Sciences of the United States of America 74:5463-5467.

583 Schier R, Marks JD, Wolf EJ, Apell G, Wong C, McCartney JE, Bookman MA, Huston JS, 584 Houston LL, Weiner LM, Adams GP. 1995. In vitro and in vivo characterization of a human 
585 anti-c-e-erbB-e single-chain $\mathrm{Fv}$ isolated from a filamentous phage antibody library.

586 Immunotechnology 1:73-81. DOI: 10.1016/1380-2933(95)00007-0

587 Shaw DE, Maragakis P, Lindorff-Larsen K, Piana S, Dror RO, Eastwood MP, Bank JA, Jumper

588 JM, Salmon JK, Yibing S, Wriggers W. 2010. Atomic-level characterization of the structural 589 dynamics of proteins. Science 330: 341-346. DOI: 10.1126/science.1187409

590 Shoichet BK, Baase WA, Kuroki R, Matthews BW. 1995. A relationship between protein 591 stability and protein function. Proceedings of the National Academy of Sciences of the United 592 States of America 92: 452-456. DOI: 10.1073/pnas.92.2.452

593 Tokuriki N, Stricher F, Serrano L, Tawfik DS. 2008. How protein stability and new functions 594 trade off. PLoS Computational Biology 4(2): e1000002. DOI: 10.1371/journal.pcbi.1000002

595 Tokuriki N, Tawfik DS. Stability effects of mutations and protein evolvability. Current Opinion 596 in Structural Biology 19: 596-604. DOI: 10.1016/j.sbi.2009.08.003

597 van den Bedem H, Bhabha G, Yan K, Wright PE, Fraser JS. 2013. Automated identification of 598 functional dynamic contact networks from X-ray crystallography. Nature Methods 10:896-902.

599 DOI: $10.1038 /$ nmeth.2592

600 Vendruscolo M, Dokholyan NV, Paci E, Karplus M. 2002. Small-world view of the amino acids 601 that play a key role in protein folding. Physical Review E 65:061910. DOI: 602 10.1103/PhysRevE.65.06191

603 Voss NR, Gerstein M. 2005. Calculation of standard atomic volumes for RNA and comparison 604 with proteins: RNA is packed more tightly. Journal of Molecular Biology 346:477-492. DOI: 605 10.1016/j.jmb.2004.11.072 
606 Williams SC, Frippiat JP, Tomlinson IM, Ignatovich O, Lefranc MP, Winter G. 1996. Sequence

607 and evolution of the human germline V $\lambda$ repertoire. Journal of Molecular Biology 264: 220-232

608 DOI: 10.1006/jmbi.1996.0636

609 Wunderlich M, Martin A, Schmid FX. 2005. Stabilization of the cold shock protein CspB from

610 Bacillus subtilis by evolutionary optimization of coulombic interactions. Journal of Molecular

611 Biology 347:1063-1076. DOI: 10.1016/j.jmb.2005.02.014

612 Wunderlich M, Schmid FX. 2006. The correlation between protein stability and dipole moment:

613 a critical test. Protein Engineering, Design and Selection 19:355-358. DOI:

614 10.1093/protein/gzl019 
615 Supplemental Figure 1. Overlapping between homologous structures. A. Sequence aligning

616 between cold shock proteins from B. subtilis (PDB entry 1CSP) and B. caldolyticus (PDB entry

617 1C9O). In green are indicated identical residues; in pink, similar residues; and in blue, sequence

618 mismatch. B. 1CSP and 1C9O structures are shown in purple and blue respectively. Despite that

619 having different thermodynamic parameters, the overlapping of cold shock proteins keeps high

620 structural similarity with a main chain RMSD of $0.5 \AA$ calculated with PyMOL software.

621

622 Supplemental Figure 2. Circular biplots. Detailed information of circular biplots indicating the

623 position of each mutation of its respective protein is shown. In Dim1 (abscissa) SDRI scores

624 were represented and in Dim2 (ordinate) thermodynamic data. Variables shown as blue vectors

625 represent the association between both variables. All plots show a positive association. A.

626 Chymotrypsin inhibitor; B. 6aJL2; C. apoflavodoxin; D. arc repressor; E. DNA-binding domain

627 of the estrogen receptor $\alpha$; F. cold shock protein from B. subtilis; G. cold shock protein from $B$.

628 caldolyticus; and H. the JAK interaction region of SOCS5.

629

630 File S1. Grade. For each residue of the protein, the number of neighbors surrounding heavy 631 atoms in a distance of $5 \AA$ was determined.

632

633 File S2. SDRI. For each residue of the protein, it is shown its solvent access, Shannon dynamical 634 entropy, RMSF, and its ranking position according to the theoretical scores.

635 
636 File S3. Experimental values from thermodynamic experiments. All experiments are

637 described in corresponding references. In Csp S, mutants marked with an asterisk (*) means that

638 the recombinant protein was analyzed with a Histidine tag.

639 File S4. Additional simulations. It is shown the RMSD, RMSF, and the hierarchy position of 640 each residue in two different conditions. In Figure R1, we show the differences between the 641 complex estrogen receptor bound with DNA after $25 \mathrm{~ns}$ molecular dynamics simulation and the 642 estrogen receptor unbound from DNA after 50 ns simulation. In Figure R2, we show the 643 convergence between simulating $25 \mathrm{~ns}$ and extending simulation to $50 \mathrm{~ns}$ of $6 \mathrm{aJL} 2$.

644 
645 Table 1. Statistics of theoretical scores - SDRIs. Skewness, kurtosis, and the normality of the

646 SDRI distribution measured with Shapiro-Wilk test. SDRI distribution of globular proteins

647 shows a good distribution unlike unstructured peptide. Analysis was performed using

648 SigmaPlot11.0 software (Systat Software, San Jose, CA).

\begin{tabular}{|c|c|c|c|}
\hline Protein & Skewness & Kurtosis & Shapiro-Wilk \\
\hline Chymotrypsin inhibitor & 0.16 & -0.59 & 0.98 \\
\hline 6aJL2 & 0.74 & -0.03 & 0.95 \\
\hline Apoflavodoxin & 0.80 & 0.12 & 0.95 \\
\hline Arc repressor & 0.51 & 0.02 & 0.97 \\
\hline Complex Estrogen/Receptor & -0.11 & -0.19 & 0.99 \\
\hline Cold shock protein BS & 0.53 & -0.67 & 0.94 \\
\hline Cold shock protein BC & 0.61 & -0.54 & 0.94 \\
\hline Unstructured peptide & 1.75 & 2.15 & 0.71 \\
\hline
\end{tabular}


650 Table 2. Statistics of SDRIs versus experimental results. Coefficient of determination $\left(\mathrm{R}^{2}\right)$

651 and Pearson correlation coefficient analysis describe mild correlation between SDRIs and 652 experimental values. Analysis was performed using SigmaPlot11.0 software (Systat Software, 653 San Jose, CA).

\begin{tabular}{|c|c|c|}
\hline Protein & $\mathbf{R}^{\mathbf{2}}$ & $\begin{array}{c}\text { Pearson Correlation } \\
\text { Coefficient }\end{array}$ \\
\hline Chymotrypsin inhibitor & 0.25 & -0.50 \\
\hline 6aJL2 & 0.22 & -0.54 \\
\hline Apoflavodoxin & 0.28 & -0.55 \\
\hline Arc repressor & 0.24 & -0.50 \\
\hline Complex Estrogen/Receptor & 0.29 & -0.54 \\
\hline Cold shock protein BS & 0.06 & -0.12 \\
\hline Cold shock protein BC & 0.08 & -0.21 \\
\hline
\end{tabular}

654 

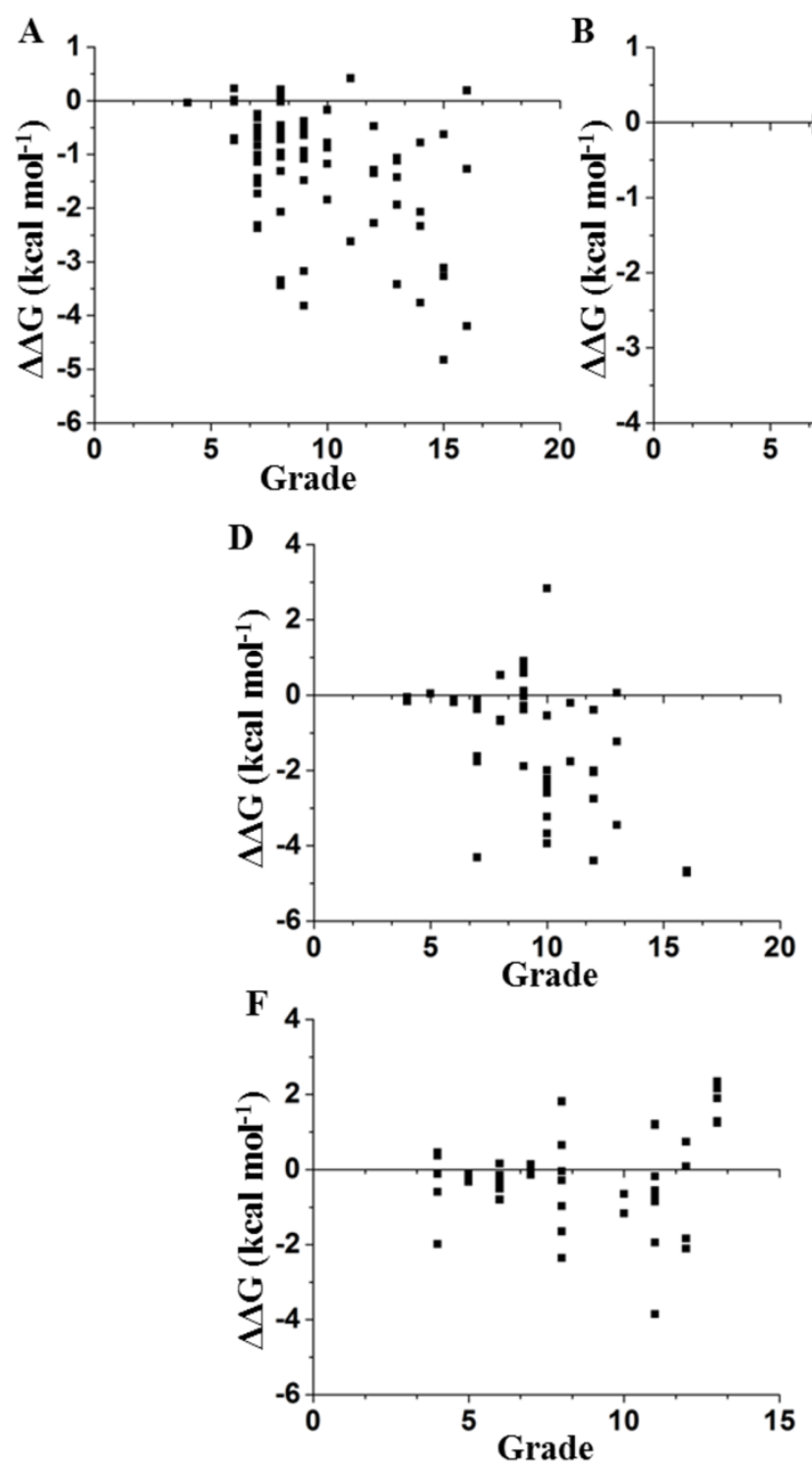
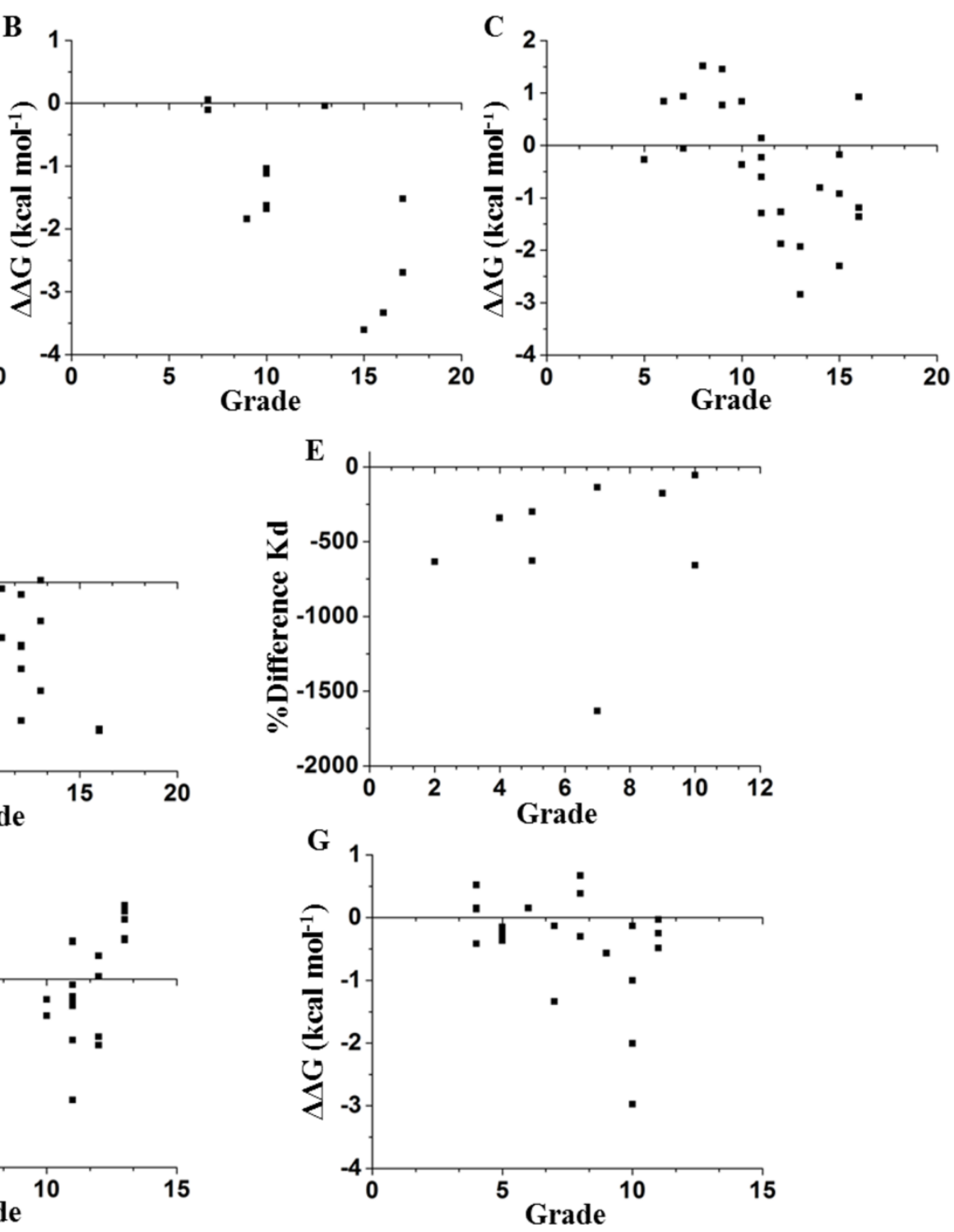

Figure 1. Contrasting of network parameter grade against experimental values. Each point

657 in the graphics represents scores determined from the number of contacts surrounding the residue

658 where the mutation was performed (see File S1). A. Chymotrypsin inhibitor; B. 6aJL2; C.

659 apoflavodoxin; D. arc repressor; E. DNA-binding domain of the estrogen receptor w; F. cold

660 shock protein from B. subtilis; and G. cold shock protein from B. caldolyticus. 


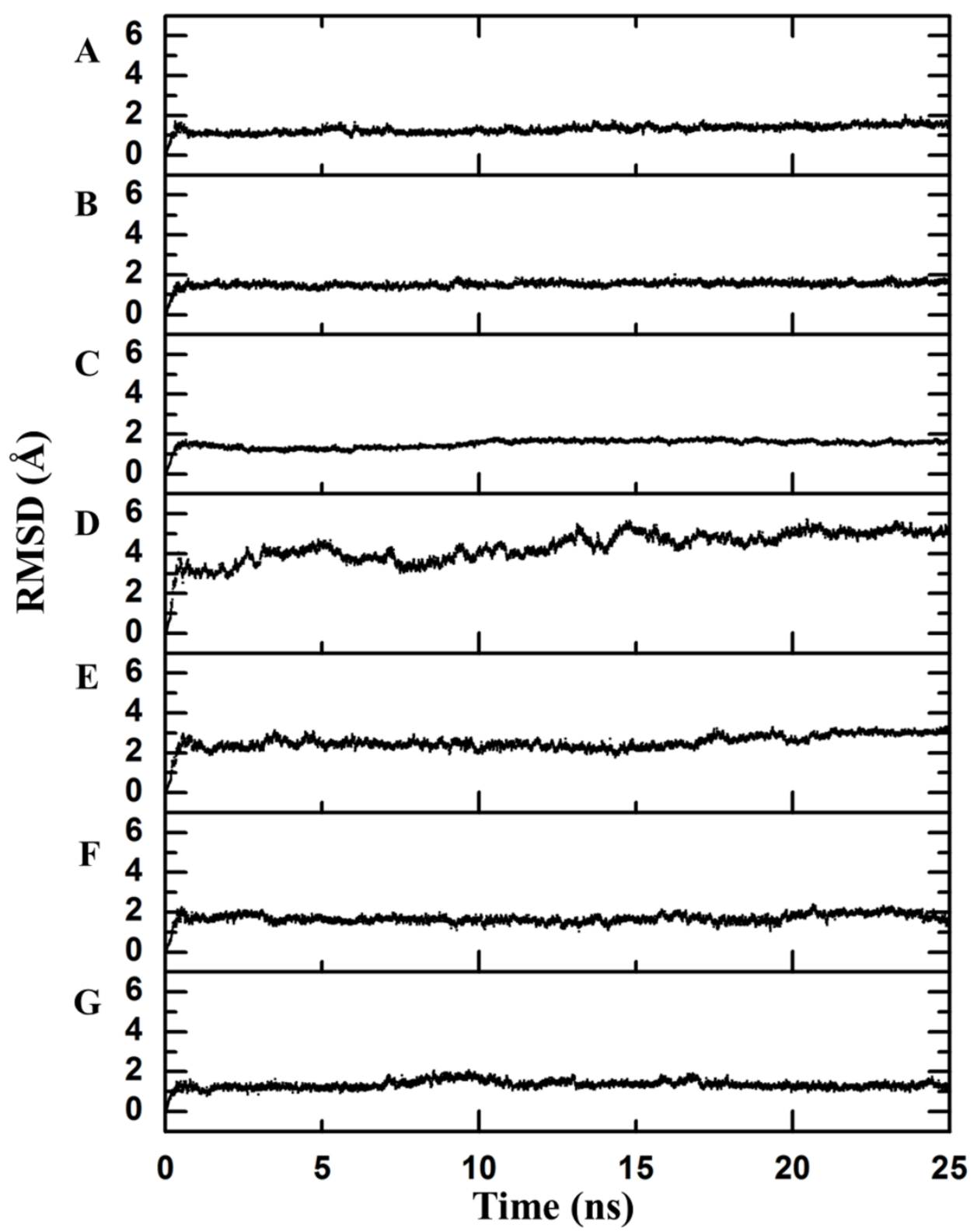

663 Figure 2. Backbone RMSD. RMSD difference between the backbone of the crystallographic

664 structure and the corresponding structure present at the indicated time of molecular dynamics;

665 this difference was calculated using Desmond. There is an observable change after the first few 666 nanoseconds of the dynamics simulations, during which the structure is "heated", but the protein 667 remains stable after that moment. A. Chymotrypsin inhibitor; B. 6aJL2; C. apoflavodoxin; D. arc 668 repressor; E. DNA-binding domain of the estrogen receptor; F. cold shock protein from $B$. 669 subtilis; and G. cold shock protein from B. caldolyticus. 

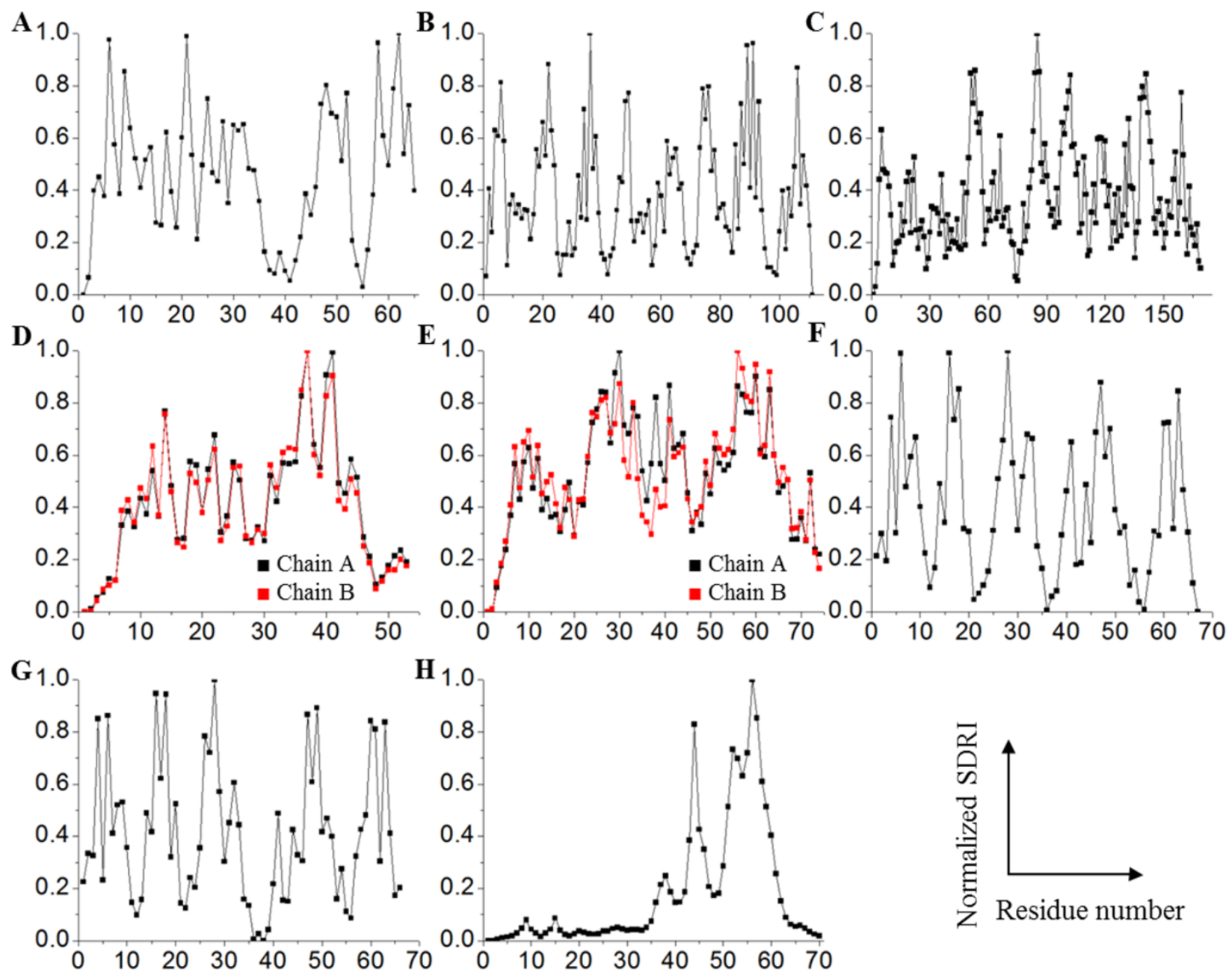

Figure 3. Contrasting of SDRIs against their corresponding sequence. Each point in the

672 graphics represents normalized SDRIs from the theoretical analysis according to their sequence

673 position (see File S2). Values near 0 indicate flexible and lesser connected residues while values

674 near 1 indicate rigid and well-connected residues: A. Chymotrypsin inhibitor; B. 6aJL2; C.

675 apoflavodoxin; D. arc repressor; E. complex of estrogen receptor $\alpha /$ DNA estrogen response

676 element; F. cold shock protein from B. subtilis; G. cold shock protein from B. caldolyticus; and

677 H. the JAK interaction region of SOCS5. 

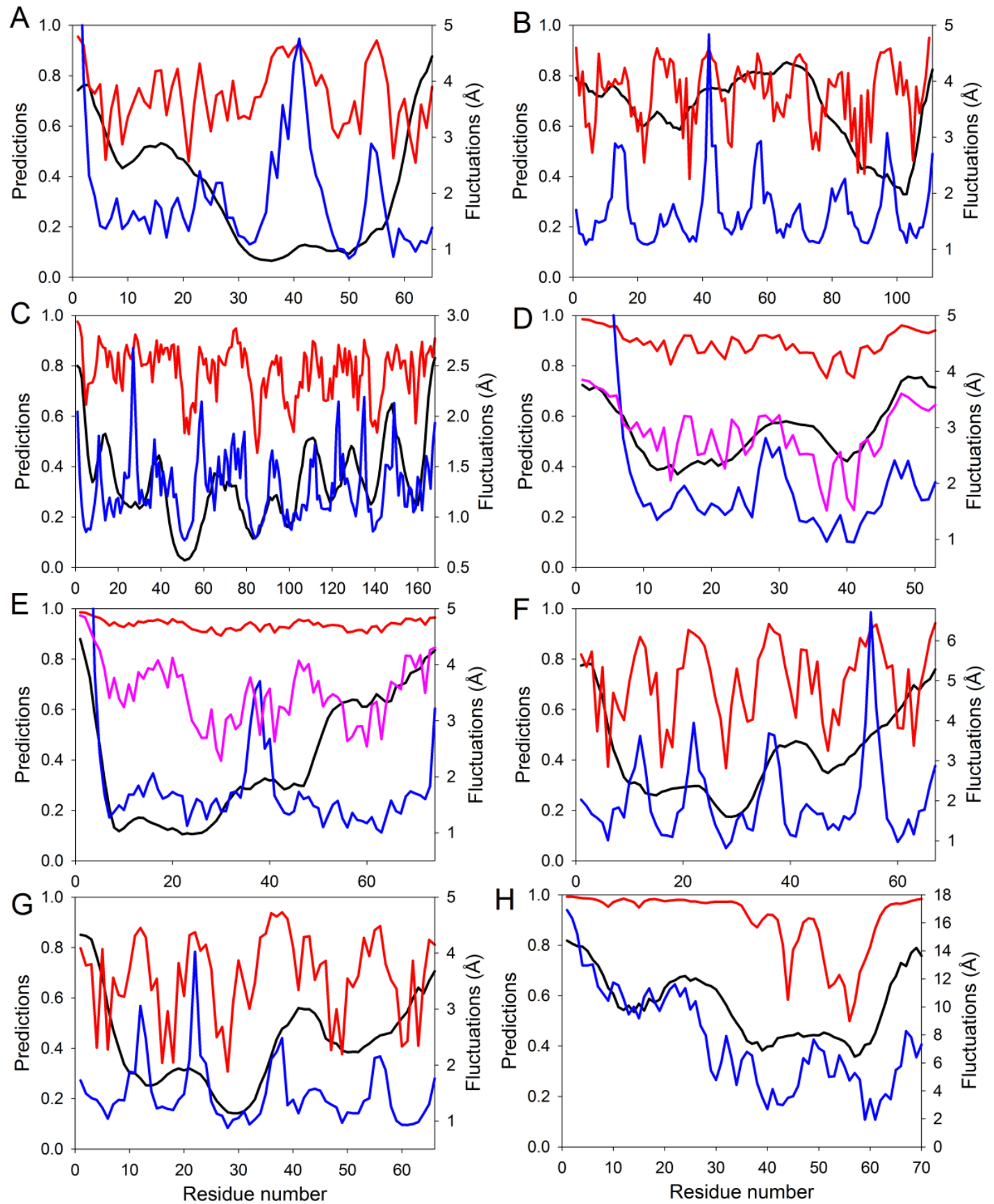

679

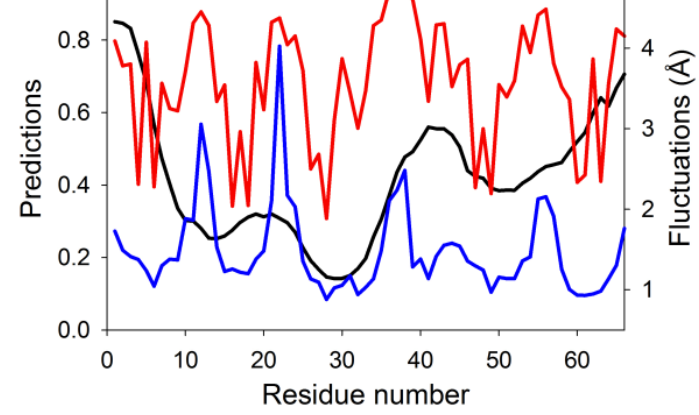

680 Figure 4. Evaluating intrinsic disorder and structural flexibility. Distributions of predicted

681 intrinsic disorder propensity evaluated by PONDR ${ }^{2}$ VSL2B (black curves), predicted structural 682 flexibility estimated by FlexPred (blue curves) and (1-SDRI) function ranking structure 683 determining residues (red or pink curves) for a set of query proteins: A. Chymotrypsin inhibitor; B. 6aJL2; C. apoflavodoxin; D. arc repressor; E. DNA-binding domain of the estrogen receptor 
$685 \alpha$; F. cold shock protein from B. subtilis; G. cold shock protein from B. caldolyticus; and H. the 686 JAK interaction region of SOCS5. Propensities for intrinsic disorder and (1- SDRI) function are 687 scaled from 0 to 1 . Since formation of two complexes - dimeric Arc repressor and a complex 688 between the estrogen receptor DNA-binding domain and the DNA estrogen response element 689 resulted in a dramatic reduction of the amplitude of the (1-SDRI) function, corresponding plots 690 (D and E) also include expanded (1- SDRI) curves shown in pink. 

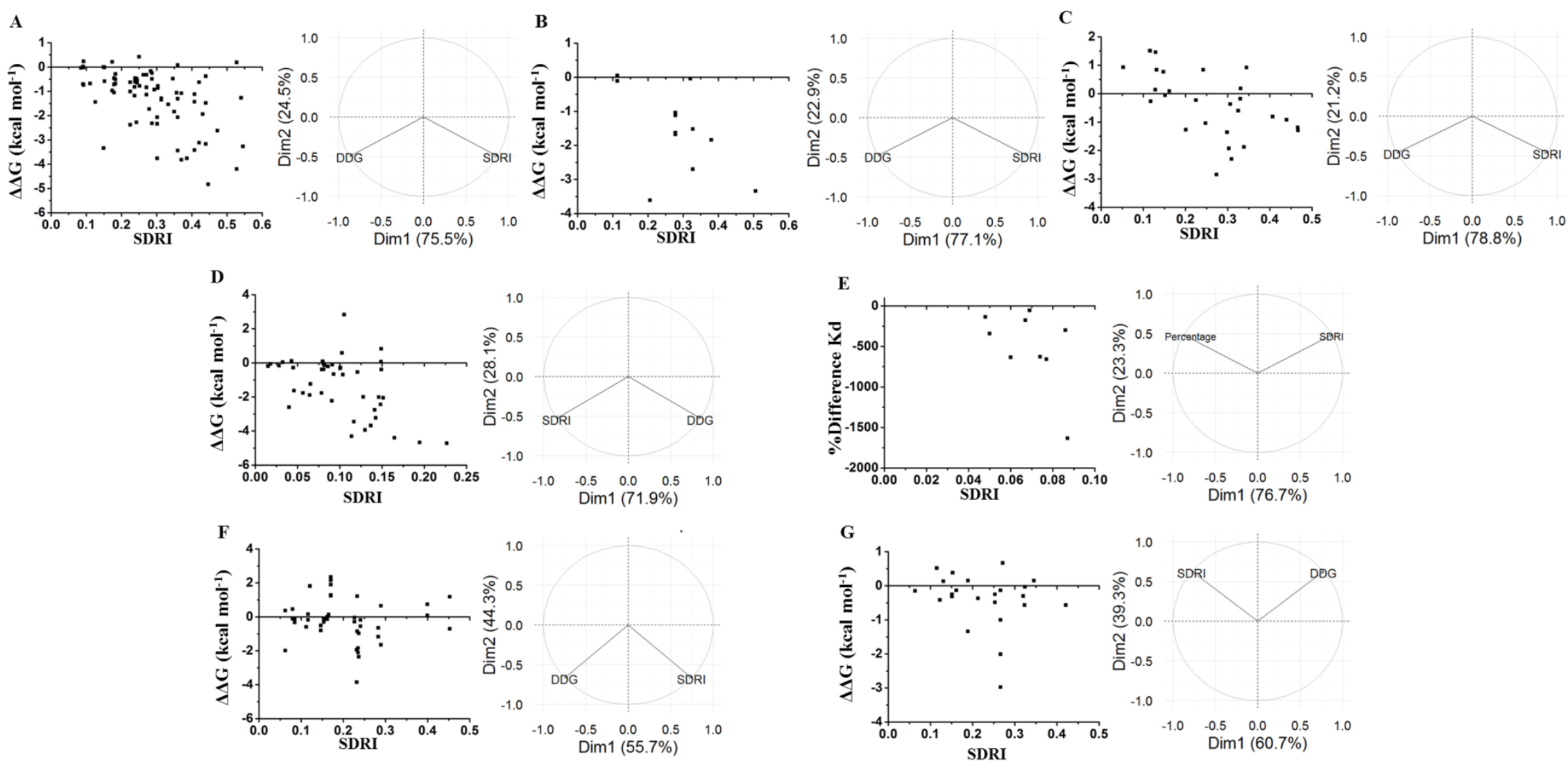

692 Figure 5. Contrasting of SDRIs against their corresponding experimental values. Each point in the graphics represents a single-

693 mutation experiment compared with the scores from the theoretical analysis (see File S3). In the case of homodimers, only the values

694 for chain A are shown. Statistical data is shown in terms of eigenvectors: DIM1 refers to SDRIs $\left(\mathrm{H}_{i} * \mathrm{RMSF}_{i}^{-1}\right)$, and DIM2 refers to the 
696 repressor; E. complex of estrogen receptor $\alpha /$ DNA estrogen response element; F. cold shock protein from B. subtilis; and G. cold 697 shock protein from B. caldolyticus. 

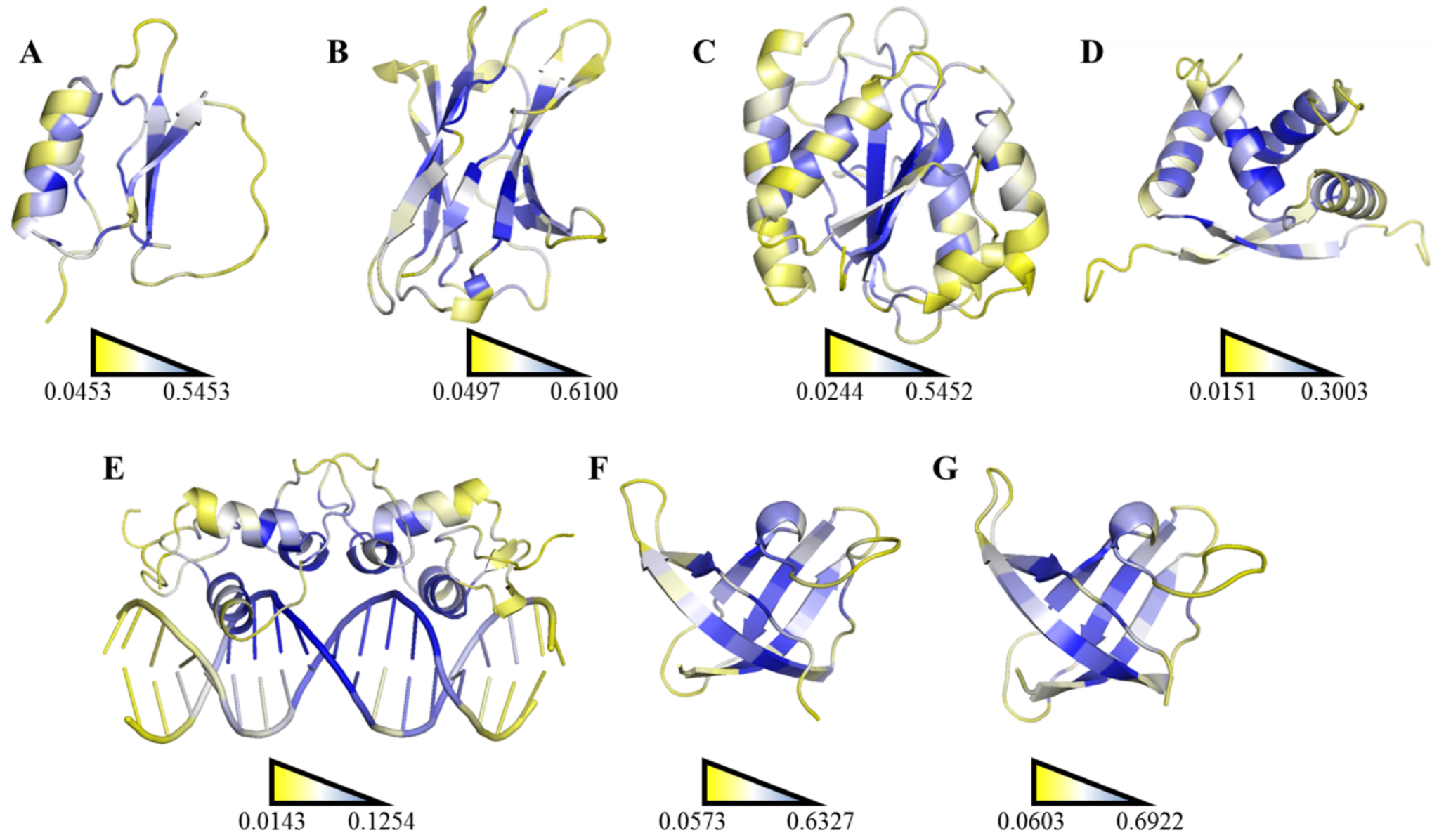

Figure 6. Ranking heat map. The highest SDRI, representing a highly connected node with restricted movement, is shown in blue,

and the lowest theoretically scored node, which is barely connected and exhibits a high degree of movement, is represented in yellow 
702 entry 2W0K); C. apoflavodoxin (PDB entry 1FTG); D. arc repressor (PDB entry 1ARR); E. complex of estrogen receptor $\alpha /$ DNA

703 estrogen response element (PDB entry 1HCQ); F. cold shock protein from B. subtilis, and G. cold shock protein from B. caldolyticus. 

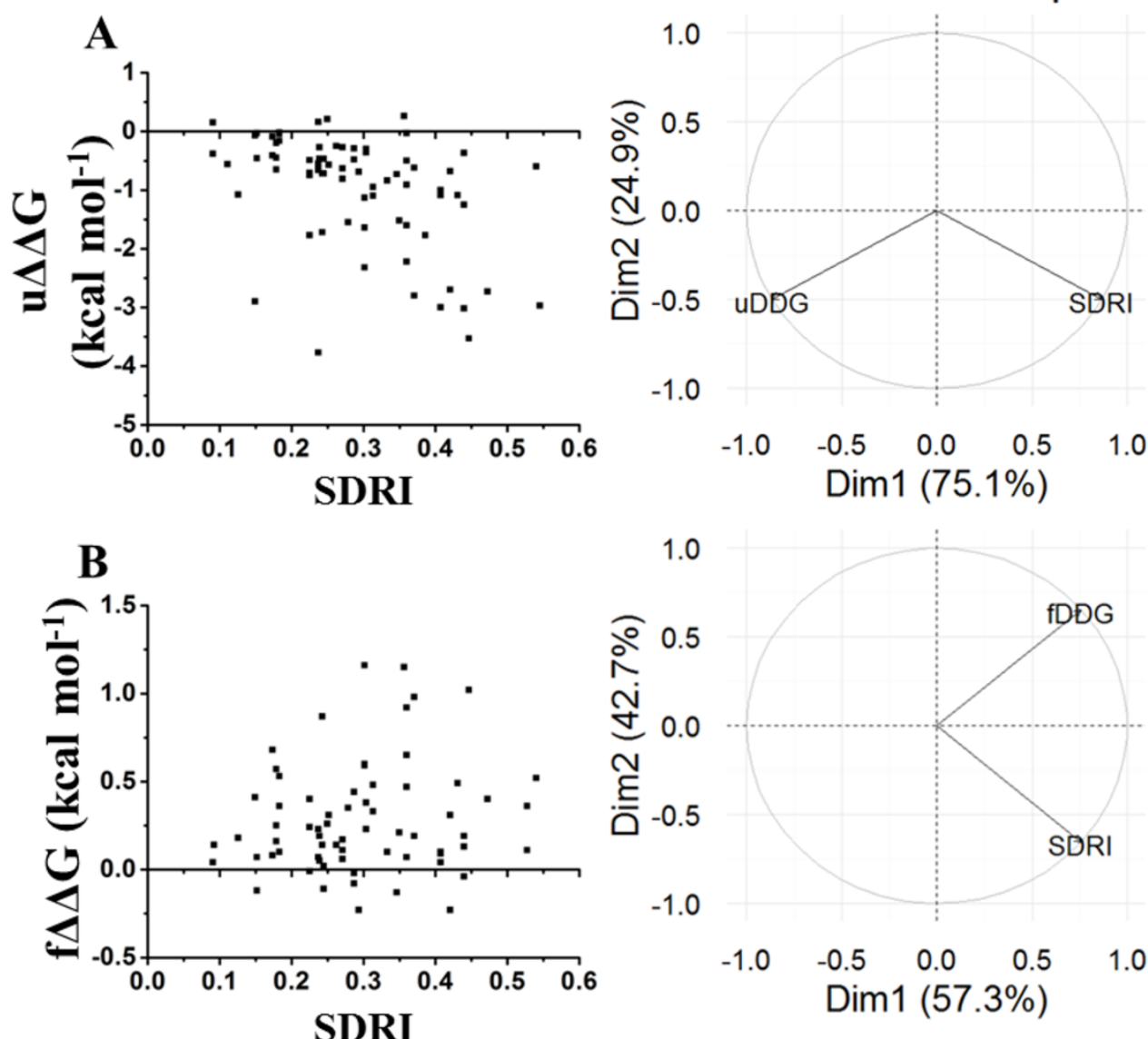
SDRI

Figure 7. Comparison of SDRIs with the kinetic unfolding and kinetic refolding processes

707 shown in circular biplots. DIM1 refers to SDRIs $\left(\mathrm{H}_{i *} \mathrm{RMSF}_{i}^{-1}\right)$, and DIM2 refers to the 708 experimental values, $\Delta \Delta \mathrm{G}(\mathrm{DDG})$ or $\mathrm{KD}$ percent difference (see File S3). A. $\Delta \Delta \mathrm{G}$ Unfolding 709 kinetics, uDDG in circular biplot; B. $\Delta \Delta \mathrm{G}$ Refolding kinetics, fDDG in circular biplot. 

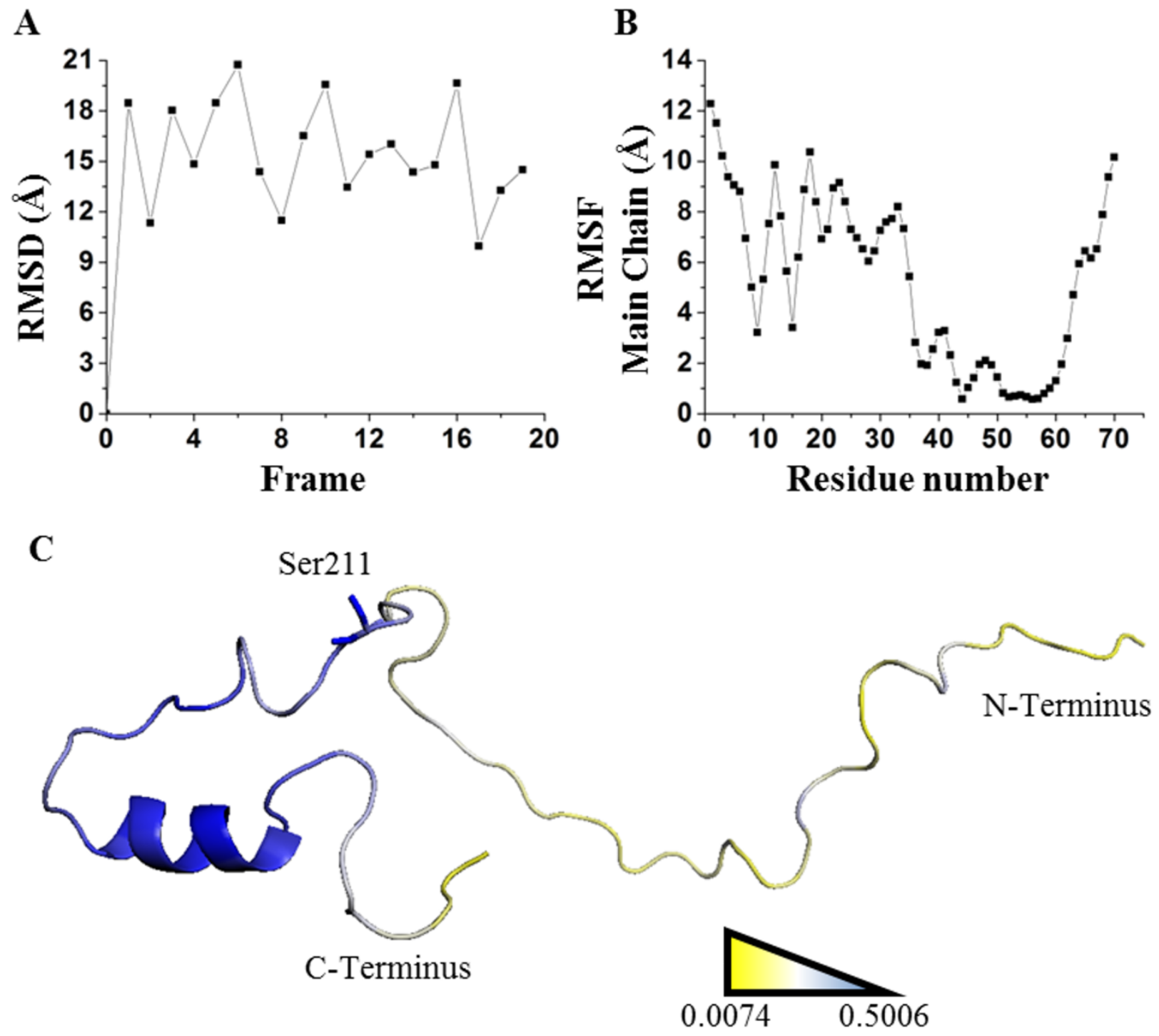

711 Figure 8. Applying SDRI approach over an unstructured peptide. Largely disordered N-

712 terminus of suppressor of cytokine signaling 5 mammalian suppressor of Janus Kinase

713 interaction region. A. RMSD values by comparing each frame against first frame from PDB

714 2N34. B. RMSF values of each residue showing that hairpin domain is more rigid than the rest of

715 the protein. C. Ranking heat map where the highest SDRI is shown in blue and the lowest SDRI 716 is represented in yellow (see File S2). 